\title{
Additions of neural monitoring for thyroid surgery
}

\author{
Hui Sun ${ }^{1} \cdot$ Gianlorenzo Dionigi $\mathbb{( I D}^{2}$
}

Received: 22 January 2018 / Accepted: 8 February 2018 / Published online: 24 February 2018

(c) Springer Science+Business Media, LLC, part of Springer Nature 2018

We read with interest the papers by B. Wojtczak et al. published on Endocrine [1, 2].

Intraoperative neural monitoring (IONM) in thyroid surgery is aimed at either identifying functional structures which cannot be recognized purely on the basis of anatomical landmarks, continuously assess the functional integrity of neural pathways which can be injured during surgery and elucidate mechanism of injury $[1,2]$.

We appreciate the authors' efforts to examine their IONM experience and the results are of interest for all clinicians involved in surgical management of thyroid diseases $[1,2]$.

Wojtczak' work should not be limited to the clinical consequences of the IONM use [1,2].

De facto, IONM has made significant additions to the knowledge of anatomy, neurophysiology and pathology of the recurrent (RLN) and external branch of the superior laryngeal nerve (EBSLN) [3].

Present and future research on IONM is aimed to optimize nerve control during thyroid dissection (Wojtczak' results) and further to close the interval between IONM results and pre- and postoperative assessment of vocal cord (VC) movement, voice quality, breathing and swallowing disorders as the Authors advocate in their paper [1, 2]. Quality of voice, breathing, and swallowing represent the final asset of the vagal nerve (VN), RLN and EBSLN system. RLN and EBSLN structures and nerve function are intimately related [3]. Intact RLN function is the

Gianlorenzo Dionigi

gdionigi@unime.it

1 Jilin Provincial Key Laboratory of Surgical Translational Medicine, China-Japan Union Hospital of Jilin University, Division of Thyroid Surgery, Changchun city, Jilin Province, China

2 Division for Endocrine and Minimally Invasive Surgery, Department of Human Pathology in Adulthood and Childhood "G. Barresi”, University Hospital G. Martino, University of Messina, Via C. Valeria 1, 98125 Messina, Italy prerequisite of intact $\mathrm{VC}$ function. Intraoperative assessment of VN and RLN function, may not be identical with VC movement and quality of voice [3].

Future research may tend to analyze the interval between the neurophysiological intraoperative results obtained by IONM during thyroid surgery and the final result at the postoperative laryngoscopy, quality of the voice indexes, and swallowing evaluation.

Translating robust monitoring research evidence to clinical practice is essential for IONM safe interpretation.

Techniques like IONM, improvements in surgical anatomy, neurophysiologist multidisciplinary enable the endocrine surgeon to detect this complex nerve system (VNRLN-EBSLN) [3]. The complex neuroanatomy and physiology requires a highly standardized IONM technique combined with an electrophysiological and clinical interpretation of the results after surgery [3].

\section{Compliance with ethical standards}

Conflict of interest The authors declare that they have no conflict of interest.

\section{References}

1. B. Wojtczak, K. Kaliszewski, K. Sutkowski, M. Bolanowski, M. Barczyński, A functional assessment of anatomical variants of the recurrent laryngeal nerve during thyroidectomies using neuromonitoring. Endocrine 59(1), 82-89 (2018). https://doi.org/10.1007/ s12020-017-1466-3

2. B. Wojtczak, K. Sutkowski, K. Kaliszewski, M. Barczyński, M. Bolanowski, Thyroid reoperation using intraoperative neuromonitoring. Endocrine 58(3), 458-466 (2017)

3. M. Barczyński, G.W. Randolph, C.R. Cernea, H. Dralle, G. Dionigi, P.F. Alesina, R. Mihai, C. Finck, D. Lombardi, D.M. Hartl, A. Miyauchi, J. Serpell, S. Snyder, E. Volpi, G. Woodson, J.L. Kraimps, A.N. Hisham, International Neural Monitoring Study Group, External branch of the superior laryngeal nerve monitoring during thyroid and parathyroid surgery: International Neural Monitoring Study Group standards guideline statement. Laryngoscope 123(Suppl 4), S1-14 (2013) 